American Journal of Environmental Sciences 8 (3): 262-270, 2012

ISSN 1553-345X

(C) 2012 Science Publications

\title{
Hospital Water Supply as a Potential Source of Opportunistic Pathogens
}

\author{
${ }^{1}$ El-Zanfaly, H.T., ${ }^{1}$ N.A. Hassanean, \\ ${ }^{2}$ E.M. Hassan and ${ }^{2}$ L.I. El-Seadawy \\ ${ }^{1}$ Department of Water Pollution Control, \\ National Research Center, Dokki, Cairo, Egypt \\ ${ }^{2}$ Department of Microbiology, Faculty of Medicine for Girls, \\ Al-Azhar University, Nasr City, Cairo, Egypt
}

\begin{abstract}
In Egypt as well as in many other developing countries, there are no specific standards for hospital water. Even water is free from the traditional bacterial indicators, it may represent a source of health hazards especially for elderly, children and patients under dialysis due to the presence of opportunistic pathogenic bacteria. The study was carried out on the bacteriological water quality at the intakes as well as the end of water treatment train of two water treatment plants that supplying three hospitals located in Greater Cairo, Egypt with water that used for different purposes. Samples of the raw water supply for the two water treatment plants (Nile River water) showed ranges of $10^{2}$ $10^{5} \mathrm{cfu} \mathrm{mL}^{-1}, 10^{2}-10^{4}$ MPN $100 \mathrm{~mL}^{-1}, 10^{2}-10^{4} \mathrm{MPN} 100 \mathrm{~mL}^{-1}$ and $10^{2}-10^{3} \mathrm{MPN} 100 \mathrm{~mL}^{-1}$ for Heterotrophic Plate Count (HPC) bacteria, Total Coliforms (TC), Fecal Coliforms (FC) and Fecal Streptococci (FS), respectively. Treated water showed considerable reduction in HPC while the other bacterial indicators reached the undetectable level. The distribution system impact on treated water quality was limited to causing an increase in HPC bacteria. A study was carried out to determine the presence of Pseudomonas aeuginosa, Aeromonas spp. and Staphylococcus aureus in hospitals tap water, water reservoirs, as well as water for preparation of hemodialysis fluids. Although the post-chlorinated water in both water treatment plants was free from bacterial indicators, it still contaminated with the three studied opportunistic pathogenic bacteria. The detected opportunistic pathogens may be attributed to the distribution system condition and/or the presence of storage tanks. Hemodialysis water samples showed the higher percentage of $P$. aeruginosa isolates which represent a major source of health risk to patient's attending dialysis process in hospitals and clinics. The presence of opportunistic bacteria in drinking water and dialysate with absence of coliform and low HPC suggested that the criteria for drinking water are usually not adequate for water used in hospitals. The purpose for which the water is to be used determines the criteria for water quality. Storage of water should be as hygienic as possible.
\end{abstract}

Key words: Opportunistic pathogens, Heterotrophic Plate Count (HPC), Fecal Streptococci (FS), Total Coliforms (TC), Fecal Coliforms (FC), distribution system, hospital water

\section{INTRODUCTION}

Opportunistic pathogens include those organisms which exist as a part of the normal body microflora but under certain conditions, they cause diseases in compromised hosts. They ordinarily are not invasive but dependent on opportunities through breaks in the natural body barriers, such as wounds, burns, depressed gastric acidity. The exposure risk includes persons who have become debilitated by some other bacterial, viral or other diseases (e.g., gastrointestinal or hepatic disease, hypo-or achlorhydria, diabetes), usually focusing on senior citizens in rest homes or newborns and infants without established natural immunity systems. The exposure risk also includes patients of all ages receiving antibiotics or chemotherapy and particularly of concern to AIDS victims (Zagainova et al., 2010).

Opportunistic pathogen infections are a serious public health problem in any areas where large numbers of people are in close confinement, particularly hospitals and senior care facilities. At least five percent of patients admitted to hospitals acquire nosocomial infections and about one percent of the affected patients

Corresponding Author: El-Zanfaly, H.T., Department of Water Pollution Control, National Research Center, Dokki, Cairo, Egypt 
die as a direct result. Many of these organisms are found among the diverse flora of water supply (Grabinska-Loniewska et al., 2007). Identification of bacteria isolated from distributed water indicated that over $30 \%$ of the total bacterial populations were opportunistic pathogens (LeChevallier et al., 1980).

1 densities in most treated municipal water supplies are generally below $100 \mathrm{cfu} \mathrm{mL}^{-1}$ except at static work locations in large housing projects, high-rise office buildings, hotels and public buildings as a result of infrequent demand as well as continued use of storage tanks, especially at warm ambient temperatures (Barrell et al., 2000; WHO, 2002).

In case of special need users (hospitals, dialysis clinics) and some families who are not satisfied with taste, odor, or other need to protect them against the probability of potential health risks associated with a given municipal water supply, is attempting to further refine water quality using special treatment devices. While the applied treatment devices may be for sometimes very effective in processing the water supply, their usefulness overtime may diminish because of its limited absorbance capacity, high concentrations of undesirable pollutants and large volume of water processed. The microbiological quality of water produced by these devices is extremely variable (Reasoner et al., 1987).

One suspected problem as a result from point-ofuse carbon filters is the increase in HPC by one or two $\operatorname{logs}$ over the numbers detected in the public water supplied at the service tap. This proliferation relates to the species of organisms passing through the filter device, seasonal change in water temperatures, the presence or absence of free residual chlorine, ambient air temperatures around the unit and the service duration for a given carbon cartridge. Non-use periods overnight or for longer intervals will also provide an opportunity for continued growth of organisms colonizing carbon filters. Under no flow conditions, water temperature can increase to ambient room temperature thereby stimulating bacterial multiplication. Chlorine residuals in this situation either form byproducts overtime in the warm static water or are effectively absorbed by carbon in the unit. As a consequence, water produced from these units frequently had higher bacterial densities in morning samples (Geldreich, 1991).

Patient exposure to waterborne microorganisms in the hospital occurs while showering, bathing, drinking water, or ingestion ice. It can also occur through contact with contaminated medical equipment such as take feed bags, of flexible endoscope and respiratory equipment that have been rinsed with tap water. The hands of healthcare personnel washed using tap water can also lead to patient exposure to opportunistic pathogens (Trautmann et al., 2001; Anaissie et al., 2002).

\section{MATERIALS AND METHODS}

Water samples: Water samples were collected from 3 hospitals located in greater Cairo, Egypt (Embaba ElAm, Kasr El-Aini and Abou El-Rish) and they supplied with water from two water utilities (Embaba and ElRoda water). The two water treatment plants depend on Nile River water as a source of raw water supply. The number and sources of the collected water samples tested are presented in Table 1.

Media, reagents and methods: Media used and methods followed are according to Standard Methods for the Examination of Water and Wastewater (Eaton and Franson, 2005). It is possible to be summarized as follows:

- MPN technique was followed for both coliforms and fecal streptococci groups determination using Lauryl Sulphate Tryptose Broth and Brilliant Green Lactose Bile Broth (presumptive and confirmed tests for total coliforms) and EC broth (for fecal coliforms)

- $\quad$ Azide Dextrose broth and m-Enterococcus agar (as presumptive and confirmatory tests for fecal streptococci)

- R2A agar for Heterotrophic Plate Count (HPC) bacteria determination following the spread plate technique (Eaton and Franson, 2005)

- Isolation of opportunistic pathogens using membrane filter technique and selective media as follows:

- M-PA-D agar as selective medium for $P$. aeruginosa determination (Vicente et al., 1986),

- Ampicillin-Dextrin Agar (ADA), as selective medium for Aeromonas spp. isolation according to Havelaar et al. (1987)

- Aeromonas hydrophila (AH) selective medium (Kaper et al., 1979)

- Mannitol-Salt Agar (MSA) for staphylococci isolation (Eaton and Franson, 2005).

- Study on $P$. aeruginosa isolates for identification according to Vicente et al. (1986) by the following tests:

- Detection and enhancement of pyocyanin production in King's a medium (King et al., 1954),

- Gelatin liquefaction (ASFM, 1981)

- Gluconate Oxidation (Carpenter, 1961) 
Am. J. Environ. Sci., 8 (3): 262-270, 2012

Table 1: Number and source of the collected water samples

\begin{tabular}{|c|c|c|c|c|c|}
\hline Place & Raw & $\begin{array}{l}\text { Post } \\
\text { chlorinated }\end{array}$ & Tap & Reservoir & Hemodialysis* \\
\hline Embaba Water Utility & 19 & 19 & - & 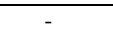 & 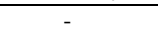 \\
\hline Embaba El-Am Hospital & - & 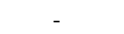 & 19 & 19 & 19 \\
\hline El-Roda Water Utility & 19 & 19 & - & - & - \\
\hline Kasr El-Aini Hospital & - & - & 19 & - & 19 \\
\hline Abou El-Rish Hospital & - & - & - & 19 & \\
\hline
\end{tabular}

*Water used to prepare the dialysis fluid

Table 2: Tests used for differentiation of Aeromonas species

\begin{tabular}{llll}
\hline Test & A. hydrophila & A. sobria & A. caviae \\
\hline Esculin hydrolysis & + & - & + \\
Lysine decarboxylase & + & + & - \\
VP test & + & + & - \\
Arabinose (Acid) & + & $-/+$ & + \\
Glucose (gas) & + & + & - \\
\hline
\end{tabular}

- Milk agar (Brown and Foster, 1970) to detect protease production

- Growth in Brain Heart Infusion Broth at $42^{\circ} \mathrm{C}$

- Oxidation / Fermentation (O/F) test according to Hugh and Leifson (1953)

- Cytochrome Oxidase test

- Arginine dihydrolase test (Moller, 1955)/(P. aeruginosa is: Oxidase + , acid production from glucose aerobically + , growth at $42^{\circ} \mathrm{C}+$, pyocyanin pigment production + , gluconate oxidizer, arginine -, liquefy gelatin +), Study on Aeromonas spp. isolates:

- A-Identification

- Suspected colonies on ADA medium (yellow colonies) were verified as Aeromonas spp. according to Havelaar et al. (1987) in two steps:

- Screening for Aeromonas hydrophila (AH) medium (Kaper et al., 1979) by stabbing in the medium and incubation at $30^{\circ} \mathrm{C}$ for $24-48 \mathrm{~h}$. Aeromonas gives an alkaline reaction (purple at the top) and acid (yellow) butt

- Biochemical identification for Aeromonas positive on the AH medium by streaking on tryptic soy agar (TSA) and identified as Aeromonas spp. according to the following reactions: Oxidase test $(+), \mathrm{O} / \mathrm{F}(+/+)$, arginine dihydrolase $(+)$, ornothine decarboxylase $(-)$, resistance to vibriostatic agent 2, 4-diamino-6, 7-diisopropylpteridone phosphate (+). Aeromonas spp differentiation of species according to Havelaar et al. (1992) (Table 2)

- Study on Staphylococci isolates:

- Identification: The suspected colonies on MSA were confirmed as Staph. aureus according to tests in Eaton and Franson (2005) as Catalase (+), Morphology and Gram staining, fermentation of glucose aerobically and anaerobically (form acid but no gas) and fermentation of mannitol aerobically (acid. and no gas)

\section{RESULTS}

The results of bacteriological analysis for water samples are illustrated in Table 3. The results of different bacteriological parameter for raw water samples from different locations studied showed non-significant differences $(p>0.05)$. There was a remarkable decrease in bacteriological parameters densities resulted from water treatment processes. The HPC determined for water samples from the two water treatment plants under investigation showed highly significant and significance differences, $(\mathrm{p}<$ 0.01 and $\mathrm{p}<0.05$ ) (Table 3).

Hospitals water samples showed negative results for bacterial indicators. Deterioration of water quality through the distribution system appeared from the high records for HPC of Embaba El-Am as well as Kasr El-Aini hospitals water samples compared by the records of the water treatment effluent. Also, hemodialysis water samples had HPC higher than the records of tap water samples. No impact resulted from a reservoir on the HPC count of EmbabalEl Am water samples, while it showed a drastic effect in case of Abou El-Rish (Table 4).

Comparing the results of the opportunistic pathogens at the two water treatment plant intakes showed that Aeromonas spp. and Staphylococci densities are higher at El-Roda than Embaba with significant difference $(\mathrm{p}<0.05)$. On the other hand, $P$. aeruginosa densities of both water treatment plants are nearly similar ( $\mathrm{p}>0.05$ ).

Number of positive P. aeruginosa samples (11) at Embaba intake was higher than that at El-Roda intake (7) and the opposite for Aeromonas spp. (Table 5).

Opportunistic pathogenic bacteria showed high resistance towards the chlorine dose used in water treatment plants $(5.0 \mathrm{ppm})$ with free residual 1.5 $2.0 \mathrm{ppm}$ in case of Embaba utility and $6-7 \mathrm{ppm}$ with free residual $1.7-2.3 \mathrm{ppm}$ in case of El-Roda utility). At El-Roda water treatment plant, postchlorination resulted in reduction in the number of positive $P$. aeruginosa samples from 7.0 to 2.0, Aeromonas spp from 11 to $7 / 0$ and Staphylococci from 7.0 to 5.0. The outlet of the two water treatment plants showed different results regarding the number of positive samples for the three opportunistic pathogenic bacteria under study (Table 5). 
Am. J. Environ. Sci., 8 (3): 262-270, 2012

Table 3: Ranges of heterotrophic plate count and bacterial indicators densities for raw Nile river and treated water samples from two water treatment plants

\begin{tabular}{|c|c|c|c|c|}
\hline \multirow[b]{2}{*}{ Test } & \multicolumn{2}{|c|}{ Embaba water treatment plant } & \multicolumn{2}{|c|}{ El-Roda water treatment plant } \\
\hline & $\begin{array}{l}\text { Raw water } \\
\text { (19) }\end{array}$ & $\begin{array}{l}\text { Treated water } \\
\text { (19) }\end{array}$ & $\begin{array}{l}\text { Raw water } \\
\text { (19) }\end{array}$ & $\begin{array}{l}\text { Treated water } \\
\text { (19) }\end{array}$ \\
\hline \multicolumn{5}{|l|}{ HPC as cfu at: } \\
\hline $28^{\circ} \mathrm{C}$ for 3 days & $\begin{array}{l}5 \times 10^{3}-2 \times 10^{5} / \mathrm{mL} \\
(39368.4 \pm 15033.6)\end{array}$ & $\begin{array}{l}<1-18 / 100 \mathrm{~mL} \\
\left(8.8 \pm 6.2^{*}\right)\end{array}$ & $\begin{array}{l}7 \times 10^{3}-1 \times 10^{5} / \mathrm{ml} \\
(35400.0 \pm 11791.3)\end{array}$ & $\begin{array}{l}<1-13 / 100 \mathrm{~mL} \\
(5.8 \pm 4.5)\end{array}$ \\
\hline $18^{\circ} \mathrm{C}$ for 7 days & $\begin{array}{l}1 \times 10^{4}-3 \times 10^{5} / \mathrm{mL} \\
(103684.2 \pm 78966.2)\end{array}$ & $\begin{array}{l}2-31 / 100 \mathrm{~mL} \\
(15.4 \pm 10.4 * *)\end{array}$ & $\begin{array}{l}1.5 \times 10^{4}-2.5 \times 10^{5} / \mathrm{ml} \\
(110842.1 \pm 60338.1)\end{array}$ & $\begin{array}{l}3-21 / 100 \mathrm{~mL} \\
(8.1 \pm 5.4)\end{array}$ \\
\hline \multicolumn{5}{|l|}{ MPN/100 ml for: } \\
\hline \multirow[t]{2}{*}{$\mathrm{TC}$} & $4.3 \times 10^{2}-6.4 \times 10^{4}$ & $<1$ & $4.6 \times 10^{2}-4.6 \times 10^{4}$ & $<1$ \\
\hline & $(18538.4 \pm 13515.1)$ & - & $(21322.1 \pm 18223.9)$ & - \\
\hline \multirow[t]{2}{*}{ FC } & $1.5 \times 10^{2}-1.1 \times 10^{4}$ & $<1$ & $2.3 \times 10^{2}-1.1 \times 10^{4}$ & $<1$ \\
\hline & $(3105.7 \pm 1843.6)$ & - & $(2803.1 \pm 1962.6)$ & - \\
\hline \multirow[t]{2}{*}{ FS } & $1.5 \times 10^{2}-3.9 \times 10^{3}$ & $<1$ & $1.5 \times 10^{2}-3.9 \times 10^{3}$ & $<1$ \\
\hline & $(1909.0 \pm 1288.0)$ & - & $(1942.6 \pm 1308.7)$ & - \\
\hline
\end{tabular}

HPC: Heterotrophic plate count TC: Total coliforms FC: Fecal coliforms FS: Fecal streptococci < 1: Not detectable * : Significant difference (p < $0.05) * *$ : Highly significant difference $(\mathrm{p}<0.01)$

Table 4: Ranges of heterotrophic plate count (HPC) for treated and hospital water samples

\begin{tabular}{|c|c|c|c|}
\hline \multirow[b]{2}{*}{ Water samples } & \multicolumn{2}{|c|}{ HPC (cfu/100 mL) at: } & \multirow{2}{*}{$\begin{array}{l}\text { Bacterial indicators } \\
\text { (TC, FC, FS) }\end{array}$} \\
\hline & $28^{\circ} \mathrm{C}$ for 3 days & $18^{\circ} \mathrm{C}$ for 7 days & \\
\hline Treated water samples from: & & & \\
\hline Embaba water treatment plant (19) & $\begin{array}{l}<1-18 \\
\left(8.8 \pm 6.2^{*}\right) \\
<1-13\end{array}$ & $\begin{array}{l}2-31 \\
\left(15.4 \pm 10.4^{* *}\right) \\
3-21\end{array}$ & \\
\hline El-Roda water treatment plant (19) & $(5.8 \pm 4.5)$ & $(8.1 \pm 5.4)$ & ND \\
\hline Hospital tap water samples: & $4-33$ & $6-40$ & \\
\hline Embaba El-Am hospital (19) & $\begin{array}{l}(19.6 \pm 9.9 * *) \\
2-28\end{array}$ & $\begin{array}{l}(23.2 \pm 11.1 *) \\
4-20\end{array}$ & \\
\hline Kasr El-Aini hospital (19) & $(12.1 \pm 8.4 * *)$ & $\left(11.7 \pm 5.2^{*}\right)$ & ND \\
\hline Hospital reservoir water samples: & $8-37$ & $9-44$ & \\
\hline Embaba El-Am hospital (19) & $\begin{array}{l}(21.5 \pm 9.8) \\
3037\end{array}$ & $\begin{array}{l}(24.3 \pm 12.0) \\
5-33\end{array}$ & \\
\hline Abou El-Rish hospital (19) & $(20.6 \pm 11.0 * *)$ & $(20.8 \pm 9.8 * *)$ & ND \\
\hline Hemodialysis water samples: & $<1-56$ & $2-60$ & \\
\hline Embaba El-Am Hospital (19) & $\begin{array}{l}\left(24.6 \pm 16.8^{* *}\right) \\
<1-25\end{array}$ & $\begin{array}{l}(24.5 \pm 19.8 *) \\
<1-26\end{array}$ & \\
\hline Kasr El-Aini Hospital (19) & $(13.8 \pm 8.3 * *)$ & $(13.8 \pm 8.3 * *)$ & ND \\
\hline
\end{tabular}

ND: Not Detected $(<1) *$ Significant difference $(\mathrm{p}<0.05) * *$ highly significant difference $(\mathrm{p}<0.01)$

Table 5: Opportunistic pathogens density (count/100 mL) in positive water samples from the two water treatment plants

\begin{tabular}{|c|c|c|c|c|c|c|c|c|c|c|c|c|}
\hline \multirow[b]{3}{*}{$\begin{array}{l}\text { Sample } \\
\text { number }\end{array}$} & \multicolumn{6}{|c|}{ Embaba water treatment plant } & \multicolumn{6}{|c|}{ El-Roda water treatment plant } \\
\hline & \multicolumn{3}{|l|}{ Raw water } & \multicolumn{3}{|c|}{ Treated water } & \multicolumn{3}{|c|}{ Raw water } & \multicolumn{3}{|c|}{ Treated water } \\
\hline & $\begin{array}{c}\text { Ps. } \\
\text { Aeruginosa }\end{array}$ & $\begin{array}{l}\text { Aeromonas } \\
\text { sp. }\end{array}$ & $\begin{array}{l}\text { Staph. } \\
\text { aureus }\end{array}$ & $\begin{array}{c}\text { Ps. } \\
\text { aeruginosa } \mathrm{s}\end{array}$ & $\begin{array}{l}\text { Aeromona } \\
\mathrm{sp}\end{array}$ & $\begin{array}{l}\text { Staph. } \\
\text { aureus }\end{array}$ & $\begin{array}{c}\text { Ps. } \\
\text { aeruginosa }\end{array}$ & $\begin{array}{l}\text { Aeromona } \\
a \quad \mathrm{sp}\end{array}$ & $\begin{array}{l}\text { Staph. } \\
\text { aureus }\end{array}$ & $\begin{array}{c}P s . \\
\text { aeruginosa }\end{array}$ & $\begin{array}{l}\text { Aeromonas } \\
\text { sp. }\end{array}$ & $\begin{array}{l}\text { Staph. } \\
\text { aureus }\end{array}$ \\
\hline 1 & $<1$ & $<1$ & $<1$ & $<1$ & $<1$ & $<1$ & 8 & 6 & $<1$ & $<1$ & $<1$ & $<1$ \\
\hline 2 & $<1$ & $<1$ & $<1$ & $<1$ & $<1$ & $<1$ & 12 & 17 & 12 & $<1$ & 5 & 4 \\
\hline 3 & 3 & $<1$ & $<1$ & 1 & $<1$ & $<1$ & 7 & 6 & $<1$ & 1 & $<1$ & $<1$ \\
\hline 4 & $<1$ & $<1$ & $<1$ & $<1$ & $<1$ & $<1$ & 3 & 8 & $<1$ & $<1$ & 2 & $<1$ \\
\hline 5 & $<1$ & $<1$ & $<1$ & $<1$ & $<1$ & $<1$ & 6 & 8 & 9 & 2 & $<1$ & 5 \\
\hline 6 & 5 & $<1$ & $<1$ & 3 & $<1$ & $<1$ & 6 & 10 & 7 & $<1$ & 7 & 3 \\
\hline 7 & 7 & 7 & 5 & 4 & 2 & 2 & $<1$ & 15 & $<1$ & $<1$ & 8 & $<1$ \\
\hline 8 & 8 & $<1$ & $<1$ & 2 & $<1$ & $<1$ & $<1$ & 17 & $<1$ & $<1$ & 9 & $<1$ \\
\hline 9 & 11 & $<1$ & 3 & 3 & $<1$ & 1 & $<1$ & $<1$ & $<1$ & $<1$ & $<1$ & $<1$ \\
\hline 10 & 9 & 8 & $<1$ & 4 & 2 & $<1$ & $<1$ & 20 & 13 & $<1$ & 13 & 8 \\
\hline 11 & 5 & $<1$ & 2 & 5 & $<1$ & $<1$ & $<1$ & 10 & 11 & $<1$ & 5 & 4 \\
\hline 12 & 8 & 10 & 6 & 6 & 4 & 3 & $<1$ & 7 & $<1$ & $<1$ & $<1$ & $<1$ \\
\hline 13 & 11 & 7 & 8 & 5 & 3 & 5 & 2 & $<1$ & 5 & $<1$ & $<1$ & $<1$ \\
\hline 14 & 6 & 9 & 6 & 6 & 4 & 4 & $<1$ & $<1$ & $<1$ & $<1$ & $<1$ & $<1$ \\
\hline 15 & 5 & 10 & 5 & 2 & 3 & 2 & $<1$ & $<1$ & 4 & $<1$ & $<1$ & $<1$ \\
\hline
\end{tabular}


Am. J. Environ. Sci., 8 (3): 262-270, 2012

Table 6: Opportunistic pathogens density (count/100 $\mathrm{mL}$ ) found in positive water samples collected from Embab water treatment plant and Embaba El- Am hospital

\begin{tabular}{|c|c|c|c|c|c|c|c|c|c|c|c|c|}
\hline \multirow[b]{3}{*}{$\begin{array}{l}\text { Samples } \\
\text { Number }\end{array}$} & \multicolumn{6}{|c|}{ Embaba water treatment plant } & \multicolumn{6}{|c|}{ Embaba El-Am hospital water } \\
\hline & \multicolumn{3}{|c|}{ Treated water } & \multicolumn{3}{|l|}{ Tap water } & \multicolumn{3}{|c|}{ Reservoir water } & \multicolumn{3}{|c|}{ Hemodialysis water } \\
\hline & $\begin{array}{c}\text { Ps. } \\
\text { Aeruginosa }\end{array}$ & $\begin{array}{l}\text { Aeromona } \\
\text { sp. }\end{array}$ & $\begin{array}{l}\text { Staph. } \\
\text { aureus a }\end{array}$ & $\begin{array}{c}\text { Ps. } \\
\text { aeruginosa }\end{array}$ & $\begin{array}{l}\text { Aeromonas } \\
t \mathrm{sp}\end{array}$ & $\begin{array}{l}\text { Staph. } \\
\text { aureus }\end{array}$ & $\begin{array}{c}\text { Ps } \\
\text { aeruginosa } \\
\end{array}$ & $\begin{array}{l}\text { Aeromonas } \\
\text { sp }\end{array}$ & $\begin{array}{l}\text { Staph. } \\
\text { aureus ae }\end{array}$ & $\begin{array}{cc}\text { Ps. } & A e \\
\text { aeruginosa } & \mathrm{sp} \\
\end{array}$ & $\begin{array}{l}\text { Aeromonas } \\
\text { sp. }\end{array}$ & $\begin{array}{l}\text { Staph. } \\
\text { aureus }\end{array}$ \\
\hline$\overline{3}$ & 1 & $<1$ & $<<1$ & $<<1$ & $<1$ & $<<1$ & $<<1$ & $<1$ & $<1$ & 11 & $<<1$ & $<<1$ \\
\hline 6 & 3 & $<1$ & $<1$ & $<1$ & $<1$ & $<1$ & $<1$ & $<1$ & $<1$ & 16 & $<1$ & $<1$ \\
\hline 7 & 4 & 2 & 2 & $<1$ & $<1$ & $<1$ & $<1$ & 5 & 3 & 17 & $<1$ & $<1$ \\
\hline 8 & 2 & $<1$ & $<1$ & $<1$ & $<1$ & $<1$ & $<1$ & $<1$ & $<1$ & 20 & $<1$ & $<1$ \\
\hline 9 & 3 & $<1$ & 1 & $<1$ & $<1$ & $<1$ & 5 & $<1$ & 8 & 22 & $<1$ & $<1$ \\
\hline 10 & 4 & 2 & $<1$ & 7 & $<1$ & $<1$ & 9 & 7 & $<1$ & 37 & $<1$ & $<1$ \\
\hline 11 & 5 & $<1$ & $<1$ & 6 & $<1$ & $<1$ & 6 & $<1$ & $<1$ & 23 & $<1$ & $<1$ \\
\hline 12 & 6 & 4 & 3 & 10 & 5 & 6 & 9 & 11 & 8 & 32 & $<1$ & $<1$ \\
\hline 13 & 5 & 3 & 5 & 5 & 7 & 8 & 8 & 8 & 10 & 37 & $<1$ & $<1$ \\
\hline 14 & 6 & 4 & 4 & 8 & 8 & 6 & 7 & 9 & 11 & 22 & $<1$ & $<1$ \\
\hline 15 & 2 & 3 & 2 & $<1$ & $<1$ & $<1$ & $<1$ & 10 & 4 & 24 & $<1$ & $<1$ \\
\hline 17 & $<1$ & $<1$ & $<1$ & $<1$ & $<1$ & $<1$ & $<1$ & $<1$ & $<1$ & 13 & $<1$ & $<1$ \\
\hline 18 & $<1$ & $<1$ & $<1$ & $<1$ & $<1$ & $<1$ & $<1$ & $<1$ & $<1$ & 11 & $<1$ & $<1$ \\
\hline + Samples & s 11 & 6 & 6 & 5 & 3 & 3 & 6 & 6 & 6 & 13 & 0 & 0 \\
\hline $\begin{array}{l}\text { Count } \\
\text { ranges } \\
\text { Mean } \pm S D\end{array}$ & $\begin{array}{l}1-6 \\
(3.7 \pm 1.6)\end{array}$ & $\begin{array}{l}2-4 \\
(3.0 \pm 0.8)\end{array}$ & $\begin{array}{l}1-5 \\
(2.8 \pm 1.3)\end{array}$ & $\begin{array}{l}5-10 \\
\quad(2.7 \pm 1.7\end{array}$ & $\begin{array}{cc} & 5-8 \\
7 * *) & (6.6 \pm 1.2)\end{array}$ & $\begin{array}{l}6-8 \\
(6.6 \pm 0.9\end{array}$ & $\begin{array}{ll} & 5-9 \\
9) & (7.3 \pm 1.5)\end{array}$ & $\begin{array}{l}5-11 \\
(8.3 \pm 1.9)\end{array}$ & $\begin{array}{l}3-11 \\
(7.3 \pm 2.9)\end{array}$ & $\begin{array}{ll} & 11-37 \\
.9) & (22.3 \pm 8.688\end{array}$ & 8) & - \\
\hline
\end{tabular}

Table 7: Opportunistic pathogens density (count/100 mL) found in positive treated water samples collected from El-Roda water treatment plant and two hospitals

\begin{tabular}{|c|c|c|c|c|c|c|c|c|c|c|c|c|}
\hline \multirow[b]{3}{*}{$\begin{array}{l}\text { Samples } \\
\text { number }\end{array}$} & \multicolumn{4}{|c|}{ El-Roda water treatment plant } & \multicolumn{4}{|c|}{ Kasr El-Aini hospital } & \multicolumn{4}{|c|}{ Abou El-Rish hospital } \\
\hline & \multicolumn{3}{|c|}{ Treated water } & \multicolumn{3}{|l|}{ Tap water } & \multicolumn{3}{|c|}{ Hemodialysis water } & \multicolumn{3}{|c|}{ Hemodialysis water } \\
\hline & $\begin{array}{l}\text { Ps. } \\
\text { Aeruginosa }\end{array}$ & $\begin{array}{l}\text { Aeromonas } \\
\text { sp. }\end{array}$ & $\begin{array}{l}\text { Staph. } \\
\text { aureus }\end{array}$ & $\begin{array}{l}\text { Ps. } \\
\text { aeruginosa } \mathrm{s}\end{array}$ & $\begin{array}{l}\text { Aeromonas } \\
\mathrm{sp}\end{array}$ & $\begin{array}{l}\text { Staph. } \\
\text { aureus }\end{array}$ & $\begin{array}{l}\text { Ps. } \\
\text { aeruginosa }\end{array}$ & $\begin{array}{l}\text { Aeromonas } \\
\mathrm{sp}\end{array}$ & $\begin{array}{l}\text { Staph. } \\
\text { aureus }\end{array}$ & $\begin{array}{c}\text { Ps. } \\
\text { aeruginosa }\end{array}$ & $\begin{array}{l}\text { Aeromonas } \\
\text { sp. }\end{array}$ & $\begin{array}{l}\text { Staph. } \\
\text { aureus }\end{array}$ \\
\hline 1 & $<1$ & $<1$ & $<1$ &, 1 & $<1$ & $<1$ & 13 & $<1$ & $<1$ & $<1$ & 7 & $<1$ \\
\hline 2 & $<1$ & 5 & 4 & $<1$ & 4 & $<1$ & 16 & $<1$ & $<1$ & $<1$ & 14 & 13 \\
\hline 3 & 1 & $<1$ & $<1$ & $<1$ & 9 & $<1$ & 12 & $<1$ & $<1$ & $<1$ & 22 & $<1$ \\
\hline 4 & $<1$ & 2 & $<1$ & $<1$ & $<1$ & $<1$ & 9 & $<1$ & $<1$ & $<1$ & 12 & 7 \\
\hline 5 & 2 & $<1$ & 5 & $<1$ & 6 & $<1$ & 9 & $<1$ & $<1$ & $<1$ & 8 & $<1$ \\
\hline 6 & $<1$ & 7 & 3 & $<1$ & $<1$ & $<1$ & 7 & $<1$ & $<1$ & $<1$ & 7 & 12 \\
\hline 7 & $<1$ & 8 & $<1$ & $<1$ & $<1$ & $<1$ & 8 & $<1$ & $<1$ & $<1$ & 9 & 15 \\
\hline 8 & $<1$ & 9 & $<1$ & $<1$ & $<1$ & $<1$ & 5 & $<1$ & $<1$ & $<1$ & 13 & 14 \\
\hline 9 & $<1$ & $<1$ & $<1$ & $<1$ & 3 & $<1$ & $<1$ & $<1$ & $<1$ & $<1$ & 11 & $<1$ \\
\hline 10 & $<1$ & 13 & 8 & $<1$ & $<1$ & $<1$ & $<1$ & $<1$ & $<1$ & $<1$ & 9 & 17 \\
\hline 11 & $<1$ & 5 & 4 & $<1$ & $<1$ & $<1$ & $<1$ & $<1$ & $<1$ & $<1$ & $<1$ & 9 \\
\hline Samples+ & 2 & 7 & 5 & 0 & 4 & 0 & 8 & 0 & 0 & 0 & 10 & 7 \\
\hline $\begin{array}{l}\text { Count } \\
\text { ranges }\end{array}$ & $1-2$ & $2-13$ & $3-8$ & - & $3-9$ & - & $5-16$ & - & - & - & $7-22$ & $7-17$ \\
\hline Mean \pm SD & $(1.5 \pm 0.5)$ & $(7.0 \pm 3.2)$ & $(4.8 \pm 1.7$ & ) $-(5.5 \pm 2.3)$ & 3) - & $(9.8 \pm 3.3)$ & - & - & - & $(11.2 \pm 4$ & $2 * *)-$ & $2.4 \pm 3.2 * *)$ \\
\hline
\end{tabular}

Table 6 and 7 showed the densities of opportunistic pathogens in the treated water supplied from the two water treatment plants and the changes within the three hospitals under study. Reservoir water samples from Abou El-Rish hospital showed the high density range of Staphylococci. Generally, three water samples (No. 12, $13,14)$ that collected from a tap and reservoir of Embaba El-Am hospital, gave evidence for the presence of the three opportunistic pathogenic bacteria under study (Table 6).
Thirty- two hospitals water supply samples showed positive results for $P$. aeruginosa, 21 of them originated from the hemodialysis units water supply (13 and 8.0 from Embaba and Kasr El-Aini hospital, respectively). The other 11 positive samples were detected in Embaba El-Am hospital water (Table 8).

Aeromonas spp. was isolated mainly from reservoir water samples (10) from Abou El-Rish and 6 of Embaba hospital), while in case of tap water, positive samples were reduced to 7 ( 4 from Kasr El-Aini and 3 from Embaba hospitals). 
Am. J. Environ. Sci., 8 (3): 262-270, 2012

Table 8: Opportunistic pathogen isolates from the two water treatment plants and the three hospitals

\begin{tabular}{|c|c|c|c|}
\hline \multirow[b]{2}{*}{ Source } & \multicolumn{3}{|c|}{ No of isolates belong to opportunistic pathogens } \\
\hline & P. aeruginosa & Aeromonas spp. & S. aureus \\
\hline \multicolumn{4}{|c|}{ Embaba water treatment plant: } \\
\hline Raw water (19) & 11 & 6 & 7 \\
\hline Treated water (19) & 11 & 6 & 6 \\
\hline \multicolumn{4}{|l|}{ Embaba El-Am hospital: } \\
\hline Tap water (19) & 5 & 3 & 3 \\
\hline Reservoir water (19) & 6 & 6 & 6 \\
\hline Hemodialysis water (19) & 13 & 0 & 0 \\
\hline \multicolumn{4}{|c|}{ El-Roda water treatment plant: } \\
\hline Raw water (19) & 7 & 11 & 7 \\
\hline Treated water (19) & 2 & 7 & 5 \\
\hline \multicolumn{4}{|l|}{ Kasr El-Aini hospital: } \\
\hline Tap water (19) & 0 & 4 & 0 \\
\hline Hemodialysis water (19) & 8 & 0 & 0 \\
\hline \multicolumn{4}{|l|}{ Abou El-Rish hospital: } \\
\hline Reservoir water (19) & 0 & 10 & 7 \\
\hline
\end{tabular}

Table 9: Differentiation of the 23 Aeromonas isolates from hospitals

\begin{tabular}{|c|c|c|c|c|c|c|c|c|}
\hline \multirow[b]{3}{*}{ Source } & \multicolumn{8}{|c|}{ Aeromonas isolates } \\
\hline & \multicolumn{2}{|c|}{ A. hydrophila } & \multicolumn{2}{|c|}{ A. Sobria } & \multicolumn{2}{|c|}{ A. caviae } & \multicolumn{2}{|c|}{ Total } \\
\hline & No. & $(\%)$ & No. & $(\%)$ & No. & $(\%)$ & No. & $(\%)$ \\
\hline \multicolumn{9}{|l|}{ Embaba El-Am hospital: } \\
\hline Reservoir water & 4 & 66.7 & 2 & 33.3 & - & - & 6 & 66.7 \\
\hline Tap water & 2 & 66.7 & - & - & 1 & 33.3 & 3 & 33.3 \\
\hline Total & 6 & 66.7 & 2 & 22.2 & 1 & 11.1 & 9 & 39.1 \\
\hline Abou El-Rish hospital: & & & & & & & & \\
\hline $\begin{array}{l}\text { Reservoir water } \\
\text { Kasr El-Aini hospital: }\end{array}$ & 7 & 70.0 & 1 & 10.0 & 2 & 20.0 & 10 & 43.5 \\
\hline Tap water & 2 & 50.0 & 2 & 50.0 & - & - & 4 & 17.4 \\
\hline Total & 15 & 65.2 & 5 & 21.7 & 3 & 13.1 & 23 & 100 \\
\hline
\end{tabular}

Table 10: Identification of the staphylocoiccal isolates from hospitals water samples

\begin{tabular}{|c|c|c|c|c|c|}
\hline \multirow[b]{2}{*}{ Test } & \multicolumn{5}{|c|}{ Embaba El-Am hospital } \\
\hline & $\begin{array}{l}\text { Tap water } \\
\text { (6) }\end{array}$ & $\begin{array}{l}\text { Reservoir } \\
(10)\end{array}$ & $\begin{array}{l}\text { Kasr El-Aini } \\
\text { tap water } \\
\text { (3) }\end{array}$ & $\begin{array}{l}\text { Abou El-Rish } \\
\text { reservoir } \\
(16)\end{array}$ & $\begin{array}{l}\text { Total } \\
\text { (35) }\end{array}$ \\
\hline Catalase & $6 / 6$ & $10 / 10$ & $3 / 3$ & $16 / 16$ & $35 / 35$ \\
\hline Microscopic examination & $5 / 6$ & $8 / 10$ & $2 / 3$ & $12 / 16$ & $27 / 35$ \\
\hline \multicolumn{6}{|l|}{ Acid from Glucose: } \\
\hline Aerobically & $4 / 6$ & $9 / 10$ & $3 / 3$ & $9 / 16$ & $25 / 35$ \\
\hline Anaerobically & $3 / 6$ & $6 / 10$ & $0 / 3$ & $7 / 16$ & $16 / 35$ \\
\hline \multicolumn{6}{|l|}{ Acid from Mannitol: } \\
\hline Aerobically & $3 / 6$ & $6 / 10$ & $0 / 3$ & $7 / 16$ & $16 / 35$ \\
\hline Coagulase & $3 / 6$ & $6 / 10$ & $0 / 3$ & $7 / 16$ & $16 / 35$ \\
\hline Identified $S$. aureus & $3 / 6$ & $6 / 10$ & $0 / 3$ & $7 / 16$ & $16 / 35$ \\
\hline
\end{tabular}

Staphylococci was isolated from Embaba El-Am tap water ( 3 samples) and increased to 6 samples by passing to the reservoir (Table 8). The 23 aeromonal isolates were differentiated into their species according to Havelaar et al. (1992). The results showed that 15 isolates out of the tested $23(65.3 \%)$ were identified as Aeromonas hydrophila (the dominant species) (Table 9).

Thirty-five suspected staphylococcal isolates were isolated from hospitals water samples, (16 from Abo El-Rish hospital water reservoir and 10 from Embaba El-Am water reservoir, 6 from Embaba El-Am and 3 from Kasr El-Aini tap water samples). The characterists of these isolates and their identification appeared in Table 10.

\section{DISCUSSION}

The presence of opportunistic pathogens in hospital water supply was followed by testing the initial water source (Nile River water) and demonstrated the effect of water treatment processes on the removal of opportunistic pathogens (quality of post-chlorinated 
water), the effect of distribution system on water quality (water source for hospitals), water quality deterioration inside the hospitals (impact of the inside distribution system and storage tanks) and the possibility of health hazards through the use of water that was contaminated by opportunistic pathogenic bacteria by patients and for dialysate preparations.

The three hospitals under study receive water from two water treatment plants. The raw water (water of the water treatment plant intake) that used by Embaba plant showed a level of fecal pollution higher than El-Roda water treatment plant. The treatment train reduced the HPC population in the finished water to a level that never exceed the value of $<35 \mathrm{cfu} \mathrm{mL}^{-1}$ which complying with the Egyptian standards for drinking water $\left(<50 \mathrm{~mL}^{-1}\right)$. In addition TC, FC and FS level in the finished water was undetectable which was also complying with the Egyptian standards. Aeromonas spp. and Staph. aureus densities are higher at El-Roda water treatment intake than Embaba intake ( $\mathrm{p}<0.05)$. So, there was no correlation between the simultaneous presence of the fecal indicators and the studied opportunistic pathogens. Therefore, the absence of bacterial indicators or the low level of HPC is not a satisfactory measure of the presence of opportunistic pathogens. Regarding the water quality at the two water treatment plants outlets, the results showed that Aeromonas spp and Staph, aureus counts were higher at El-Roda $(\mathrm{P}<0.01)$ and $(\mathrm{p}<0.05)$. While the actual data showed that $P$. aeruginosa range was higher at the outlet of the Embaba plant than El-Roda. Generally, it is possible to conclude that according to opportunistic pathogens densities ranges, water quality of Embaba water treatment plant was better than El-Roda one.

Opportunistic pathogens were highly resistant to the conventional water treatment processes including the chlorine used in post-chlorination. Aeromonas spp. has also been recovered from chlorinated water (Havelaar et al., 1990). It was demonstrated by Hirata et al. (1991) that $P$. aeruginosa was more resistant to chlorine than $E$. coli, this may be due to the slimes produced by $P$. aeruginosa (Seyfried and Fraser, 1980). That may support the recommendation of using both Staphylococci and P.aeruginosa as indicators for the satisfactory of the treatment process (Kiss et al., 1983). Staphylococci were isolated from water samples free from both coliforms and streptococci (Alico and Dragonjae, 1986).

In the present study, the deterioration of water quality through the distribution system was demonstrated and confirmed by Hapcioglu et al. (2005). The deterioration includes the high counts in opportunistic pathogens. The formation of biofilm on the surface of pipelines and sloughing to the water is the explanation for the deterioration in water quality (Edwards, 2000). The released bacteria are usually chlorine resistant (LeChevallier et al., 1988). The hospital, water storage tanks represent a place for bacteria proliferation including a source for opportunistic pathogens contamination (Felfoldi et al., 2010).

The water of hemodialysis units that used for preparation of dialysis fluids at both Embaba El-Am and Kasr El-Aini hospitals represent a possible source of $P$. aeruginosa and so its proliferation together with other organisms was possible to occur. In case of Kasr El-Aini hospital, $P$. aeruginosa cannot reach the detectable level in tap water, while it was isolateed from hemodialysis water ( 8 positive out of 19 tested samples) with density range of $5.0-16 \mathrm{cfu} 100 \mathrm{~mL}^{-1}$. In this respect, Favero et al. (1974) reported that softening, deionization or reverse osmosis processes used to prepare the dialysis fluid may represent a source of bacteriological quality deterioration. They added that in the absence of residual chlorine, microorganisms can multiply in the distribution system downstream from the membrane during the Reverse Osmosis (RO) process. Also, the design of the distribution system for the treated water has a contribution in increasing the level of bacterial contamination. In several hemodialysis centers, storage tanks or dead ends in the distribution system allowed the multiplication of gramnegative bacteria ( $P$. aeruginosa in particular). Geldreich (1991) added that $P$. aeruginosa has the ability to grow prodigiously in and on RO and electrodialysis membranes.

\section{CONCLUSION}

- Although the post-chlorinated water in both water treatment plants was free from bacterial indicators, it still contaminated with the three studied opportunistic pathogens. This can be explained by the possibility of their high resistance to chlorine. Further study on virulence of opport-unistic pathogens and the effectiveeness of new disinfection methods should be encourage

- The water supply system should ensure the provision of safe water

- Hemodialysis water samples showed the higher percentage of $P$. aeruginosa isolates which represent a major source of health risk to patients attending dialysis process in hospitals and clinics

- The storage systems should be as hygienic as possible

- Emphasis on monitoring water treatment effectiveness and distribution system integrity must not focusing on the traditional bacteriological parameters of water that leaving the treatment plant 
- Water utilities need to minimize HPC through optimization the treatment steps, reducing assimilable organic carbon in distribution system and storage tanks and minimization the dead ends in the distribution system

- Rapid methods for monitoring water quality should be followed

- The presence of opportunistic bacteria in drinking water and hemodialysis water with absence of coliforms and low HPC suggested that the present standards for water quality should be reevaluated and ideal indicator or indicators are required

- In the light of high percentages of the presence of elderly, children, patients suffering from kidney failure in hospitals, the need for an additional local water treatment should be considered

\section{REFERENCES}

Alico, R.K. and M.F. Dragonjae, 1986. Evaluation of culture media for recovery of Staphylococcus aureus from swimming pools. Applied Environ. Microbiol., 51: 699-702.

Anaissie, E.J., S.R. Penzak and M.C. Dignani, 2002. The hospital water supply as a source of nosocomial infections: A plea for action. Arch. Intern. Med., 162: 1483-1492. PMID: 12090885

ASFM, 1981. Manual of Methods for General Bacteriology. 1st Edn., American Soc Fo Microbiology, Washington, D.C., ISBN-10: 0914826301, pp: 536.

Barrell, R.A., P.R. Hunter and G. Nichols, 2000. Microbiological standards for water and their relationship to health risk. Commun. Dis. Public Health, 3: 8-13. PMID: 10743312

Brown, M.R. and J.H. Foster, 1970. A simple diagnostic milk medium for Pseudomonas aeruginosa. J. Clin. Pathol., 23: 172-177. PMID: 4987392

Carpenter, K.P., 1961. The relationship of the enterobacterium A 12 (Sachs) to Shigella boydii 14. Microbiology, 26: 535-542. DOI: 10.1099/00221287-26-3-535

Eaton, A.D. and M.A.H. Franson, 2005. Standard Methods for the Examination of Water and Wastewater. 21st Edn., American Public Health Association, Washington, D.C., ISBN-10: 0875530478, pp: 1200.

Edwards, C., 2000. Problems posed by natural environments for monitoring microorganisms. Mol. Biotechnol., 15: 211-223. DOI: 10.1385/MB:15:3:211
Favero, M.S., L.A. Carson, W.W. Bond and N.J. Petersen, 1974. Factors that influence microbial contamination of fluids associated with hemodialysis machines. Applied Environ. Microbiol., 28: 822-830.

Felfoldi, T., Z. Heeger, M. Vargha and K. Marialigeti, 2010. Detection of potentially pathogenic bacteria in the drinking water distribution system of a hospital in Hungary. Clin. Microbiol. Infect., 16: 89-92. DOI: 10.1111/j.1469-0691.2009.02795.x

Geldreich, E., 1991. Opportunistic organisms and the water supply connection. J. Clin. Microbiol. Rev., 4: 101-156.

Grabinska-Loniewska, A. G. Wardzynska, E. Pajor, D. Korsak and K. Boryn, 2007. Transmission of specific groups of bacteria through water distribution system. Pol. J. Microbiol., 56: 129138. PMID: 17650683

Hapcioglu, B., Y. Yegenoglu, Z. Erturan, Y. Nakipoglu and $\mathrm{H}$. Issover, 2005. Heterotrophic bacteria and filamentous fungi isolated from a hospital water distribution system. Indoor Built Environ., 14: 487493. DOI: $10.1177 / 1420326 X 05060039$

Havelaar, A.H., F.M. Schets, A.V. Silfhoul, W.H. Jansen and G. Wieten et al., 1992. Typing of Aeromonas strains from patients with diarrhoea and from drinking water. J. Applied Microbiol., 72: 435-444. DOI: $\quad 10.1111 / \mathrm{j} .1365-$ 2672.1992.tb01857.x

Havelaar, A.H., J.F.M. Versteegh and M. During, 1990. The presence of Aeromonas in drinking water supplies in Netherlands. Zentralbl. Hyg. Umweltmet., 190: 236-256.

Havelaar, A.H., M. During and J.F. Versteegh, 1987. Ampicillin-dextrin agar medium for the enumeration of Aeromonas species in water by membrane filtration. J. Applied Microbiol., 62: 279-287. DOI: 10.1111/j.1365-2672.1987.tb02410.x

Hirata, T., K. Kawamura, S. Sonoki, K. Hirata and M. Kaneko et al., 1991. Clostridium perfringens, as an indicator microorganism for the evaluation of the effect of wastewater and sludge treatment systems. Water Sci. Technol., 24: 367-372.

Hugh, R. and E. Leifson, 1953. The taxonomic significance of fermentative versus oxidative metabolism of carbohydrates by various gram negative bacteria. J. Bacteriol., 66: 24-26.

Kaper, J. R.J. Seidler, H. Lockman and R.R. Colwell, 1979. Medium for the presumptive identification of Aeromonas hydrophila and enterobacteriaceae. Applied Environ. Microbiol., 38: 1023-1026.

King, E.O., M.K. Ward and D.E. Raney, 1954. Two simple media for the demonstration of pyocyanin and fluorescin. J. Lab. Clin. Med., 44: 301-307. PMID: 13184240 
Kiss, P., B. Lanyi, A. Bodi and Z. Forde, 1983. Incidence of Pseudomonas aeruginosa serogroups in drinking water: Use of serotyping in the control of water supplies. Acta. Microbiol. Hung., 30: 131137. PMID: 6419534

LeChevallier, M.W., C.D. Cawthon and R.G. Lee, 1988. Factors promoting survival of bacteria in chlorinated water supplies. Applied Environ. Microobiol., 54: 649-654.

LeChevallier, M.W., R.J. Seidler and T.M. Evans, 1980. Enumeration and characterization of standard plate count bacteria in chlorinated and raw water supplies. Applied Environ. Microbiol., 40: 299-239.

Moller, V., 1955. Simplified tests for some amino acid decarboxylases and for the arginine dihydrolase system. Acta Pathol. Microbiol. Scand., 36: 158-172. DOI: 10.1111/j.1699-0463.1955.tb04583.x

Reasoner, D.J., J.C. Blannon and E.E. Geldreich, 1987. Microbiological characteristics of third-faucet point-of-use devices. J. Am. Water Works Assoc., 79: 60-66.

Seyfried, P.L. and D.J. Fraser, 1980. Persistence of Pseudomonas aeruginosa in chlorinated swimming pools. Can. J. Microbiol., 26: 350-355. DOI: 10.1139/m80-057
Trautmann, M, T. Michalsky, H. Woedack, V. Radosavljevic and M. Ruhnke, 2001. Tap water colonization with Pseudomonas aeruginosa in a surgical Intensive Care Unit (ICU) and relation to Pseudomonas infections of ICU patients. Infect. Control Hosp. Epidemiol., 22: 49-52. PMID: 11198025

Vicente, A.D., J.J. Borrego, F. Arrabal and P. Romero, 1986. Comparative study of selective media for enumeration of Pseudomonas aeruginosa from water by membrane filtration. Applied Environ. Microbiol., 51: 832-840.

WHO, 2002. Guidelines on Prevention and Control of Hospital Associated Infection. 1st Edn., The Organization, New Delhi, pp: 49.

Zagainova, A.V., I.U.G. Talaeva, R.A. Dmitrieva, F.I. Ingel and V.V. Iurcheko et al., 2010. Evaluation of epidemic risk from pathogenic and opportunistic bacteria isolated from water of its various use types. Gig. Sant. 5: 68-73. PMID: 21341500 